\title{
EFFECT OF TAMSULOSIN ON STENT- RELATED SYMPTOMS; A PROSPECTIVE STUDY
}

$1,2,3$

Urology Department

Shaikh Zayed Hospital Lahore

Correspondence Address: Dr. Muhammad Muzammil Tahir Urology Department

Shaikh Zayed Hospital Lahore

dr_muzamil@yahoo.com

Article received on: 16/01/2016

Accepted for publication: 21/01/2016

Received after proof reading: 21/01/2016

\section{Dr. Rana Ata ur Rehman', Dr. Muhammad Muzammil Tahir ${ }^{2}$, Dr. Muhammad Seerwan ${ }^{3}$}

\begin{abstract}
Introduction: Cystoscopic intraluminal placement of ureteral stents has become a routine practice in urology. Ureteral stents preserve urine flow from the kidney to the bladder in cases of ureteral obstruction (intrinsic or extrincis). In patients with obvious ureteral obstruction, the placement of a ureteral stent will restart urine transport and protect the kidney from possible risks. Ureteral stents are troublesome in some patients and causes LUTS. Study Design: Prospective randomized controlled trial. Setting: Urology Department, Sheikh Zayed Hospital Lahore. Period: Six month started from August 2015 to December 2015. Material and Methods: 100 patients who were randomly divided into two equal groups. The patients were between 18 to 50 years of age of both gender undergo retrograde double-J ureteral stent placement. Before the double-J stent will be applied, all patients completed an International Prostate Symptom Score (IPSS) questionnaire for evaluation of lower urinary tract symptoms. Patients were divided in two groups on the basis of lottery method tamsolusin group (1) and placebo group (2). Tamsulosin group patients was given tamsulosin post operatively and placebo group was given a placebo postoperatively. Results: There were total 100 patients who were enrolled in this study with a mean age of $42.63 \pm 6.24$. There were $75(75 \%)$ were male while $25(25 \%)$ were female. The mean IPSS sore at presentation was $2.47 \pm 1.43$ and post treatment was 5.20 \pm 1.65 . There was significant difference in IPSS score in control group with mean $5.28 \pm 1.69$ versus study as mean $2.22 \pm 1.05$ group, $p$-value $=0.010$. Conclusion: There is difference in lowering of IPSS score in the patients who are given tamsolusin versus those who were retained on placebo.
\end{abstract}

Key words: Double J Stent, Tamsolusin, lower Urinary Tract Symptoms

Article Citation: Ata ur Rehman R, Tahir MM, Seerwan M. Effect of tamsulosin on stent-related symptoms; a prospective study. Professional Med J 2016;23(1):114-118. DOI: $10.17957 / T P M J / 16.3254$

\section{INTRODUCTION}

Cystoscopic intraluminal ureteral stents placement has become a routine practice in urology. Ureteral stents preserve urine flow from the kidney to the bladder in cases of ureteral obstruction, both intrinsic and extrinsic. In patients with obvious ureteral obstruction, the placement of a ureteral stent will restart urine transport and protect the kidney from possible risks. The stents also prevent extravasations of urine, after an operation or trauma. ${ }^{1}$

All stents may cause morbidity such as, dysuria, flank pain hematuria, infection, encrustation and migration. The ideal stent material should be radiopaque, biocompatible and relieve intraluminal and extraluminal obstruction, cause little discomfort to the patient, resist encrustation and infection and be widely available at a reasonable cost. To date, no stent material meets all of these criteria. ${ }^{2}$ Typically, stents may cause storage lower urinary tract symptoms (LUTS) and reduce health related quality of life. ${ }^{3} \mathrm{An}$ important factor of stent related symptoms is the pressure transmitted to the renal pelvis during urination and trigonal irritation due to intravesicular part of the stent. ${ }^{4}$ Although different modalities are available in an attempt to minimize the morbidity of stents, some authors postulated that the length and position have to be adequate. ${ }^{5}$ Some authors suggested that the use of selective alpha blockers and anticholinergics would be effective, while others postulated that intravesical instillation of chemical agents or periureteral injection of botulinum toxin have important role in relieving LUTS. ${ }^{6}$ Tamsulosin acts as a selective inhibitor of $\alpha-1 \mathrm{a}$ and $1-d$-mediated 
contraction of the distal ureter, bladder trigone and proximal urethral smooth muscle. It is postulated that relaxing these smooth muscles decreases bladder outlet resistance and voiding pressure, thereby decreasing renal reflux and voiding symptoms. ${ }^{7,8}$

The purpose of study is to determine the outcomes of tamsulosin in reducing stent related lower urinary tract symptoms, leading to reduce hospital stay and increase patient comfort. There is variability in the mean IPSS score in case and control as per mentioned in the published studies. Moreover, no such study has been conducted in our population. If the symptoms of urinary obstruction are less with tamsulosin (less increase in IPSS than control), then tamsulosin can be given post stenting to reduce the symptoms of obstruction.

\section{MATERIALS AND METHODS}

\section{Inclusion Criteria}

(1) All Patients 18 to 50 years of age

(2) Patients of both sex (male and female)

(3) Patients undergoing retrograde double-J ureteral stent placement.

\section{Exclusion Criteria}

(1) Age $<18$ years.

(2) Patients refusing to participate in the study.

(3) Patients, who are known allergic to tamsulosin.

(4) History of previous ureteral stenting due to false perception of symptoms.

(5) Patients with bilateral double J stenting, because of aggravation of lower urinary tract symptoms due to increased trigonal stimulation.

(6) Bladder pathology like diagnosed bladder tumour.

(7) Benign prostatic hyperplasia diagnosed on digital rectal examination.

(8) Overactive bladder (already diagnosed by urodynamics)

(9) Urinary tract infection diagnosed on urine culture and sensitivity.
After approval of hospital ethical committee, all the patients presenting in the department of urology, Shaikh Zayed hospital Lahore fulfilling the inclusion criteria were included in the study. Duration of study was from August 2015 to December 2015. An informed consent was obtained from the patients after discussion of risk versus benefit ratio with them. All patients completed an International Prostate Symptom Score (IPSS) questionnaire for evaluation of lower urinary tract symptoms before the double-J stenting. Patients were divided in two groups on the basis of lottery method, Tamsolusin ${ }^{\mathrm{R}}$ group (1) and placebo group (2). In tamsulosin group patients were given tamsulosin post operatively and placebo group were given a placebo postoperatively. Double-J stenting was done in patients under local anesthesia and intravenous sedation (dormicum, $0.05 \mathrm{mg} / \mathrm{kg}$ ). In all cases, a $4.7 \mathrm{~F}$ and depending on the patient, a 26 or $28 \mathrm{~cm}$ long polyurethane stent was placed. X-ray KUB was done to confirm that stent was properly applied.

The drug was packed in boxes and the boxes were labelled with the group numbers as" 1 " and "2". After being randomized, the patients will receive numbered boxes containing unnamed drugs and were informed about drugs' side-effects without attention to the groups' number. The participants and physicians who prescribe drugs were blinded to the type of medications. The patients were randomly assigned to receive a placebo (Iron tablet) (group 2) or 400 micrograms of tamsulosin (according to British National Formulary 67) once daily (group 1). Moreover, patients were prescribed a single dose of intravenous analgesic after surgery to relieve post-operative pain and were also received $500 \mathrm{mg}$ acetaminophen tablets for pain control during the stenting period. Based on the policy at my institution, 500 mg of ciprofloxacin was prescribed as a prophylactic antibiotic during three days after stenting.

Before stenting and at fourth week's post-operative day, the patients were asked to complete the IPSS questionnaire to assess stent-related discomforts. Mean IPSS score was calculated as per operational definition. All the data was recorded 
on predesigned proforma.

\section{RESULTS}

There were total 100 patients who were enrolled in this study with a mean age of $42.63 \pm 6.24$. There $75(75 \%)$ were male while $25(25 \%)$ were female. The mean IPSS sore at presentation was $2.47 \pm 1.43$ and post treatment was $5.20 \pm 1.65$ (Table-I).

Patients were divided into two groups, control group (number of patients 50) and study group (number of patients 50).

There was significant difference in IPSS score in control group with mean $5.28 \pm 1.69$ versus study as mean $2.22 \pm 1.05$ group, $p$-value $=0.010$ (Table -II).

\begin{tabular}{|l|c|c|}
\hline & Mean & Std. Deviation \\
\hline Post-treatment IPSS & 5.2000 & 1.65755 \\
\hline Baseline IPSS score & 2.4700 & 1.43868 \\
\hline
\end{tabular}

Table-I. Stratification for baseline IPSS score $p$-value $=0.054$

\begin{tabular}{|l|c|c|c|c|}
\hline & Group & N & Mean & Std. Deviation \\
\hline Post-treatment IPSS & Control & 50 & 5.2800 & 1.69079 \\
\cline { 2 - 5 } & Study & 50 & 2.2200 & 1.05540 \\
\hline
\end{tabular}

Table-II. Comparison of study versus control group $p$-value $=0.010$

\section{DISCUSSION}

$80 \%$ of patients is believed to be affected by stent discomfort after double $\mathrm{J}$ stentig. Patients after double $J$ stenting usually complain of various stent related symptoms, such as voiding, storage $O A B$ symptoms, pain and hematuria. These symptoms are associated with reduced health related quality of life. ${ }^{9}$

The exact pathophysiology of DJ stent related symptoms remains unknown; however there are theories that it could be due to local irritation of trigonal mucosa, which contains $\alpha$-1D receptors and lower ureteral smooth muscle spasms and bladder instability which give symptoms similar to BPH (benign prostatic hyperplasia). ${ }^{10}$ Different maneuver are adopted to overcome troublesome double $\mathrm{J}$ stent related symptoms, some reported that stent length, girth adjustment and avoiding distal end crossing the midline are essential and have important role in relieving double $\mathrm{J}$ stent related symptoms. ${ }^{11}$ Some investigators introduced a different design, incthe Tail Stent model that was having proximal $7 \mathrm{~F}$ pigtail and tapered distal end $3 \mathrm{~F}$ tail that lie in the bladder. This tapered stent was compared to standard 7F double- $J$ stents that are in routine practice. In a randomized single-blind trial involving 60 patients and showed markedly reduction in the double $\mathrm{J}$ stent related symptoms after using above mentioned DJ stent. ${ }^{12}$ However in another study conducted by Hao et al and Thomas whom showed no significant effect of length and girth on double $\mathrm{J}$ stent related symptoms. ${ }^{13,14}$ Damiano et al..$^{15}$ reported that there was no symptoms difference between stent with different size, whereas there was a tendency of small diameter stents to dislodge more often. Chew et al reported that trigonal irritation due to change in body position led to movement of distal end within the bladder and induced more and stent related symptoms. Lang and associates ${ }^{17}$ stated that a possible mechanism of relief of stent-related symptoms could be smooth muscle relaxation of lower ureter and trigone as well as reducing ureteric motility. Wang and his colleagues suggested that relaxation of bladder neck/prostatic smoothmuscle, is other possible mechanisms for control of stent-related symptoms, setting a rationale behind using alpha blockers in overcoming ureteral stent symptoms.$^{18}$ There is another theory that antimuscarinic agents have important role in the improvement of stent-related symptoms caused by detrusor over activity, that leads to involuntary bladder contraction. ${ }^{19}$

In our study it is proven that there is difference in lowering of IPSS score in the patients who are given tamsolusin versus those who were retained on placebo. Similar results were published by Wang et al in a prospective randomized study comparing tamsulosin to placebo in 79 patients using (USSQ) reported that tamsulosin improved stent related urinary symptoms, QoL, and they recommended its routine use..$^{18}$ Also Damiano et al reported that tamsulosin has a positive effect on double $\mathrm{J}$ stent related urinary symptoms. ${ }^{18}$ 
However, Kuyumcuoglu et al reported in a prospective randomized study that tamsulosin was not different than placebo in controlling double $\mathrm{J}$ stent-related symptoms. ${ }^{1}$ In my study, tamsolusin monotherapy controlled stent-related symptoms evidenced by statistically significant differences in the IPSS total score, pre- and post-stent insertion, when compared to placebo. In another study conducted by Lee et al in a prospective, randomized, and placebo-controlled study that postoperative solifenacin use was effective and well tolerated in controlling double $\mathrm{J}$ stent related symptoms..$^{20}$ Norris et al reported in a placebo controlled trial that there were no differences between oxybutynin and placebo in controlling stent-related symptoms. ${ }^{21}$ So, there is no benefit of using oxybutynin in controlling stent-related symptoms.

Kuyumcuoglu et al. reported that tolterodine SR $4 \mathrm{mg}$ has more effective role as that of anti-inflammatory and alpha blocker in controlling stent-related symptoms. In another study, conducted by Park et al reported that tolterodine was most effective and significantly able to improve pain and urinary symptom index scores when compared with alfuzosin and placebo. ${ }^{22}$ A limitation study was the lack of patient homogeneity, as we included patients with different urologic procedures. The superiority of combined tamsulosin and solifenacin therapy was reported previously by Lim and his colleagues, who reported that combined use of solifenacin and tamsulosin was significantly better than either drug alone in reducing double $\mathrm{J}$ stent related symptoms. ${ }^{23}$ In contrast, Lee et al in their prospective randomized study over 20 patients using a combination of Tamsulosin and tolterodine reported no statistically significant difference when compared to placebo, and also when combination therapy was compared to tamsulosin monotherapy, no beneficial effect was reported. ${ }^{20}$

In our opinion, LUTS related to double $\mathrm{J}$ stent placement can be overcome by the developments of new treatment strategies. Double $\mathrm{J}$ stent related symptoms might be relieved by altering the design and material of the stents. Biotechnol- ogy could help the way in this field. However, the ideal biomaterial has yet to be discovered by further investigation. It is now need of time to sort out different technique to relieve DJ stent related symptoms, whether in the form of pharmacotherapy or stent design.

\section{CONCLUSION}

There is difference in lowering of IPSS score in the patients who are given tamsolusin versus those who were retained on placebo in Patients with DJS related symptoms.

Copyright (C) 21 Jan, 2016.

\section{REFERENCES}

1. Ugur Kuyumcuoglu, MD; Bilal Eryildirim, MD; Murat Tuncer, MD; et al. Effectiveness of medical treatment in overcoming the ureteral double-J stent related symptoms. Can Urol Assoc J 2012;6:E234-237.

2. Wein, Kavoussi et al : Wein: Campbell-Walsh Urology, 10th ed 2012,1305.

3. Alastair D. Lamb, Sarah L.Vowler, Richard Johnston, Nick Dunn and Oliver J. Wiseman. Meta-analysis showing the beneficial effect of alpha blockers on ureteric stent discomfort. BJUI 2011;.1464-410X.

4. Kyoung Taek Lim, Yong Tae Kim, Tchun Yong Lee, Sung Yul Park. Effects of Tamsulosin, Solifenacin, and Combination Therapy for the Treatment of Ureteral Stent Related Discomforts. Korean J Urol 2011;52:485-488.

5. Al-Kandari AM, Al-Shaiji TF, Shaaban H, Ibrahim HM, Elshebiny $\mathrm{YH}$, Shokeir AA. Effects of proximal and distal ends of double-J ureteral stent position on postprocedural symptoms and quality of life: a randomized clinical trial. J Endourol 2007;21:698-702.

6. Gupta $\mathrm{M}$, Patel $\mathrm{T}$, Xavier $\mathrm{K}$ et al: Prospective randomized evaluation of periureteral botulinum toxin type A injection for ureteral stent pain reduction. J Urol 2010;183: 598.

7. Sun Ju Lee, Changhee Yoo, Cheol Young Oh, Yong Seong Lee, et al. Stent Position Is More Important than $\alpha$-Blockers or Anticholinergics for Stent-Related Lower Urinary Tract Symptoms after Ureteroscopic Ureterolithotomy: A Prospective Randomized Study. Korean J Urol 2010;51:636-641.

8. Dellis A, Joshi HB, Timoney AG et al: Relief of stent related symptoms: review of engineering and pharmacological solutions. J Urol 2010;184:1267.

9. R. R. Byrne, B. K. Auge, J.Kourambas,R.Munver, F. 
Delvecchio, and G. M. Preminger, "Routine ureteral stenting is not necessary after ureteroscopy and ureteropyeloscopy: a randomized trial," Journal of Endourology, vol. 16, no. 1, pp. 9-13, 2002.

10. M. Duvdevani, B. H. Chew, and J. D. Denstedt, "Minimizing symptoms in patients with ureteric stents," Current Opinion in Urology, vol. 16, no. 2, pp. 77-82, 2006.

11. Rane, A. Saleemi, D. Cahill, S. Sriprasad, N. Shrotri, and R. Tiptaft, "Have stent-related symptoms anything to do with placement technique?" Journal of Endourology, vol. 15, no. 7, pp. 741-745, 2001.

12. M.D. Dunn, A. J. Portis, S. A. Kahn et al., "Clinical effectiveness of new stent design: randomized single-blind comparison of tail and double-pigtail stents," Journal of Endourology, vol. 14, no. 2, pp. 195-202, 2000.

13. C.-H. Ho, S.-C. Chen, S.-D. Chung et al., "Determining the appropriate length of a double-pigtail ureteral stent by both stent configurations and related symptoms," Journal of Endourology, vol. 22, no. 7, pp. 1427-1431, 2008.

14. R.Thomas, "Indwelling ureteral stents: impact of material and shape on patient comfort," Journal of Endourology, vol. 7, no. 2, pp. 137-140, 1993.

15. Damiano R, Autorino R, De Sio M, Giacobbe A, Palumbo IM, D'Armiento M. Effect of Tamsulosin in Preventing Ureteral Stent- Related Morbidity: A Prospective Study. J Endourol. 2008;22:651-5.

16. B.H.Chew, B. E. Knudsen, L. Nott et al., "Pilot study of ureteral movement in stented patients: first step in understanding dynamic ureteral anatomy to improve stent comfort," Journal of Endourology, vol. 21, no. 9, pp. 1069-1075, 2007.
17. R. J. Lang, M. E. Davidson, and B. Exintaris, "Pyeloureteral motility and ureteral peristalsis: essential role of sensory nerves and endogenous prostaglandins," Experimental Physiology, vol. 87, no. 2, pp. 129-146, 2002.

19. C.-J. Wang, S.-W. Huang, and C.-H. Chang, "Effects of tamsulosin on lower urinary tract symptoms due to double-j stent: a prospective study," Urologia Internationalis, vol. 83, no. 1, pp. 66-69, 2009.

19. Agarwal, S. Dhiraaj, V. Singhal, R. Kapoor, and M. Tandon, "Comparison of efficacy of oxybutynin and tolterodine for prevention of catheter related bladder discomfort: a prospective, randomized, placebo-controlled, double-blind study," British Journal of Anaesthesia, vol. 96, no. 3, pp. 377-380, 2006.

20. Y. J. Lee, K. H. Huang, H. J. Yang, H. C. Chang, J. Chen, and T. K. Yang, "Solifenacin improves double-J stent-related symptomsin both genders following uncomplicated ureteroscopic lithotripsy," Urolithiasis, vol. 41, pp. 247-252, 2013.

21. Norris RD, Sur RL, Springhart WP, Marguet CG, Mathias $\mathrm{BJ}$, Pietrow PK, et al. A Prospective, Randomized, Double-blinded, placebo-controlled comparison of extended release oxybutynin versus phenazopyridine for the management of postoperative ureteral stent discomfort. Urology. 2008;71:792.

22. S. C. Park, S. W. Jung, J.W. Lee, and J. S. Rim, "The effects of tolterodine extended release and alfuzosin for the treatment of double-j stent-related symptoms," Journal of Endourology, vol. 23, no. 11, pp. 1913-1917, 2009.

23. K. T. Lim, Y. T. Kim, T. Y. Lee, and S. Y. Park, "Effects of tamsulosin, solifenacin, and combination therapy for the treatment of ureteral stent related discomforts," Korean Journal of Urology, vol. 52, no. 7, pp. 485-488, 2011.

AUTHORSHIP AND CONTRIBUTION DECLARATION

\begin{tabular}{|c|c|c|c|}
\hline Sr. \# & Author-s Full Name & Contribution to the paper & Author $=$ s Signature \\
\hline 1 & Dr. Rana Ata ur Rehman & Original Author & $x^{2}=$ \\
\hline 2 & Dr. M. Muzammil Tahir & Original Author & sent \\
\hline 3 & Dr. Muhammad Seerwan & Original Author & Bnan. \\
\hline
\end{tabular}

